Enhancement of light polarization from electrospun polymer fibers by room temperature nanoimprint lithography

This content has been downloaded from IOPscience. Please scroll down to see the full text. 2010 Nanotechnology 21215304

(http://iopscience.iop.org/0957-4484/21/21/215304)

View the table of contents for this issue, or go to the journal homepage for more

Download details:

IP Address: 137.99.31.134

This content was downloaded on 04/08/2015 at 06:34

Please note that terms and conditions apply. 


\title{
Enhancement of light polarization from electrospun polymer fibers by room temperature nanoimprint lithography
}

\author{
Stefano Pagliara ${ }^{1,2}$, Andrea Camposeo ${ }^{1}$, Elisa Mele ${ }^{1,3}$, \\ Luana Persano $^{1}$, Roberto Cingolani ${ }^{1,4}$ and Dario Pisignano ${ }^{1,2}$ \\ ${ }^{1}$ NNL, National Nanotechnology Laboratory of Istituto Nanoscienze-Consiglio Nazionale \\ delle Ricerche, c/o Distretto Tecnologico ISUFI, via Arnesano, I-73100 Lecce, Italy \\ ${ }^{2}$ Istituto Superiore di Formazione Interdisciplinare ISUFI, Università del Salento, \\ via Arnesano, I-73100 Lecce, Italy \\ E-mail: dario.pisignano@unisalento.it
}

Received 14 December 2009, in final form 26 March 2010

Published 30 April 2010

Online at stacks.iop.org/Nano/21/215304

\begin{abstract}
We demonstrate the enhancement of the polarization ratio of light emitted from electrospun conjugated polymer nanofibers, by means of nanoimprint lithography carried out at room temperature. We provide evidence of tailoring the polarization properties by patterning light-emitting fibers at the nanoscale. The polarization ratios are increased up to a factor of 2.4 by gratings with periodicity $(560 \mathrm{~nm})$ matching the emission wavelength of the employed conjugated polymer. The use of room temperature nanoimprint lithography to pattern light-emitting polymer nanofibers represents a strategic route for realizing photonic crystals and distributed feedback polarized emitters on one-dimensional organic nanostructures.

(Some figures in this article are in colour only in the electronic version)
\end{abstract}

\section{Introduction}

Organic one-dimensional nanostructures are attracting increasing attention for their application in a wide variety of research fields [1]. For instance, the achievement of miniaturized lightemitting sources is gathering interest in view of integration in sensors and lab-on-chips. These devices generally have to be connected to external light sources for fluorescence excitation, thus reducing portability. In addition, the small liquid volumes (down to $\mathrm{pL}$ ) and low analyte-antigen concentrations $(\sim \mathrm{nM})$ used in microfluidic devices make sensitivity a crucial issue. In these applications, the excitation intensities are usually much larger than those from the chromophore fluorescence to be detected, and optical filtering of the excitation light can generally limit sensitivity [2]. Therefore, these classes of devices would greatly benefit from the availability of miniaturized, ultimately nanoscale, polarized organic light

3 Present address: Italian Institute of Technology (I.I.T.), Center for Biomolecular Nanotechnologies, via Barsanti 1, I-73100 Arnesano (LE), Italy.

4 Present address: Italian Institute of Technology (I.I.T.), via Morego 30, I16163 Genova, Italy. sources, allowing one to easily decouple excitation and sample emission [3].

In general, obtaining miniaturization and polarization of molecular light sources still remains a challenging issue. Polarized photoluminescence (PL) is generally reported from individual organic and inorganic nanowires [4, 5] and nanorods [6], and from molecular materials oriented on substrates [7, 8] or in mesoporous silica [9]. By contrast with other nanofabrication and alignment methods, many polymers are easily processable via electrostatic spinning (ES), a simple and inexpensive technique which allows one to realize fibers with diameters from micrometers to a few nanometers and lengths up to a kilometer [10]. Electrospun nanofibers have been investigated as promising components for many applications [11-16] including passive optical devices [11] and, although generally obtained in the form of an isotropic non-woven mat, they can be aligned into arrays by suitable collectors $[11,12]$. The internal molecular orientation in electrospun fibers is also under investigation [13, 17-19]. These and other studies [3, 14-16] suggest that microscopically molecularly oriented nanofibers 
exhibit remarkable anisotropy in their optical properties, and that these structures can be employed as polarized light sources.

However, polarized emission has been early investigated only on uniaxially aligned arrays of fibers, based on poly(phenylene vinylene) [20] or polyfluorene [21] derivatives, spun on gap electrodes and eventually undergoing mechanical stretching. The measured PL polarization ratio, $\chi_{\mathrm{PL}}=\frac{\mathrm{PL}_{\|}}{\mathrm{PL}_{\perp}}$, where $\mathrm{PL}_{\|}\left(\mathrm{PL}_{\perp}\right)$ is the value of the PL intensity with the analyzer oriented parallel (perpendicular) to the array axis, ranging from the order of a few units to 25 , depending on the employed compound and processing conditions [20,21]. By contrast, the polarization performance of fluorescent, individual conjugated polymer nanofibers has not been widely explored [22]. Furthermore, since nanopatterning of conjugated polymers can influence the emission by both excitonic and photonic mechanisms, the gentle, possibly room temperature, structuring of electrospun nanofibers certainly deserves to be investigated in depth.

In this paper, we report on room temperature nanoimprint lithography (RT-NIL) as an approach for enhancing the polarization of light emitted by single, electrospun conjugated polymer nanofibers. We provide evidence of tailoring the polarization properties by patterning at the nanoscale, enhancing the optical anisotropy by imprinting periodic features longitudinally to the fiber axis. This simple method is depicted in figure 1. As-spun fibers are made of partially aligned polymer backbones due to the high applied electrostatic fields and intense flow and stretching involved in the ES process (figures 1(a) and (b)). Upon texturing fibers by suitable gratings via RT-NIL (figures 1(c) and (d)), the resulting patterned structures can present a higher degree of alignment of polymer backbones along the axis of each imprinted feature (figures 1(e) and (f)) and enhanced PL polarization. These results open the way to the fabrication of photonic crystal structures and distributed feedback polarized devices on light-emitting electrospun nanofibers.

\section{Experimental details}

\subsection{Electrostatic spinning}

The light-emitting conjugated polymer poly[(9,9-dioctylfluorenyl-2,7-diyl)-co-(1,4-benzo-\{2,1'-3\}-thiadiazole)] (F8BT, American Dye Source Inc., Quebec, Canada) used in this study has a molecular weight of about 141000 . It is dissolved in tetrahydrofuran $\left(\mathrm{C}_{4} \mathrm{H}_{8} \mathrm{O}\right.$, Fluka, Buchs, Switzerland, NJ). Polymer solutions are prepared with concentrations in the range $0.7-1.0 \mathrm{mM}$. ES is performed by placing about $0.2 \mathrm{ml}$ of solution into a $1.0 \mathrm{ml}$ plastic syringe tipped with a 27 gauge stainless steel needle. The positive lead from a high voltage supply (XRM30P, Gamma High Voltage Research Inc., Ormond Beach, FL) is connected to the metal needle applying a bias around $5 \mathrm{kV}$. The solution is injected into the needle at a constant rate of $2 \mu 1 \mathrm{~min}^{-1}$ by a syringe pump (33 Dual Syringe Pump, Harvard Apparatus Inc., Holliston, MA), which prevents dripping at the end of the metallic capillary. Two $2 \mathrm{~mm}$-thick Al electrodes, mutually separated by $1.5-2.0 \mathrm{~cm}$,
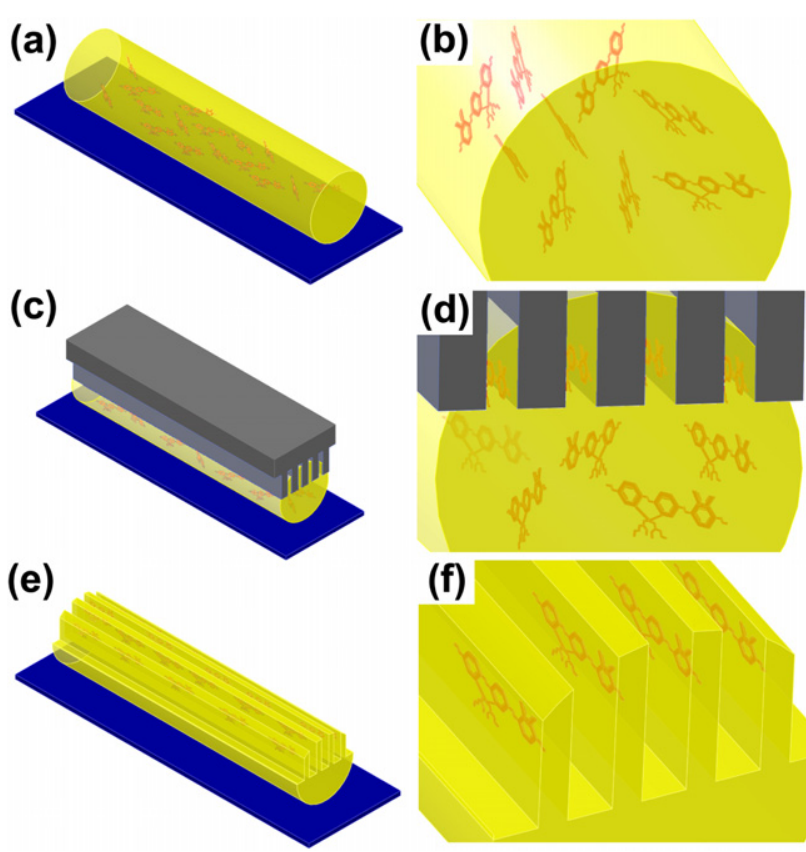

Figure 1. Scheme of the mechanism for the enhancement of PL polarization: (a) and (b) partial alignment of the polymer backbones in as-spun fiber along its longitudinal axis; (c) and (d) on-fiber RT-NIL with periodic features longitudinal to its axis; (e) and (f) enhanced alignment of polymer backbones within the patterned features.

are placed at a distance of about 5-6 $\mathrm{cm}$ from the needle and used as collectors. Free-standing fibers through the electrodes are finally collected on $1 \times 1 \mathrm{~cm}^{2}$ quartz slides for optical investigation. All the ES experiments are performed at room temperature with air humidity about $40 \%$.

\subsection{Room temperature nanoimprint lithography}

The master templates for subsequent imprinting exhibit parallel grooves with period $(\Lambda)$ of $560 \mathrm{~nm}$, depth of about $200 \mathrm{~nm}$, and features' width of about $400 \mathrm{~nm}$ over a patterned area of $1 \times 4 \mathrm{~mm}^{2}$. The period of the master is chosen in order to well-match the PL spectral region of our conjugated polymer. According to the coupling condition given by the Bragg-scattering of radiation by a distributed feedback microcavity consisting of a grating, one has $\mathbf{k}_{\mathbf{0}} \sin \Theta=$ $\pm \mathbf{k}_{\mathbf{g}} \pm n \mathbf{G}$, where $\mathbf{k}_{\mathbf{0}}$ and $\mathbf{k}_{\mathbf{g}}$ are the wavevectors of free and guided waves, respectively, $\Theta$ is the external viewing angle, $\mathbf{G}$ is the Bragg vector $(|\mathbf{G}|=2 \pi / \Lambda)$, and $n$ is an integer. The previous relation, stating the conservation of the inplane component of the radiation momentum at the patterned interface, roughly provides a period value of $560 \mathrm{~nm}$ for the here employed polymer, considering an effective refractive index of the imprinted nanostructure of about 1.8, and an almost-normal viewing direction $\left(\Theta<14^{\circ}\right)$. The masters are first exposed by electron-beam lithography with a Raith 150 system. Afterwards, $15 \mathrm{~nm}$ of $\mathrm{Cr}$ are thermally evaporated onto the substrate surface and, upon $\mathrm{Cr}$ lift-off in acetone, the masters undergo an $\mathrm{Ar}$ and $\mathrm{CF}_{4}$ plasma for reactive ion etching. The resulting depth of the features relates to the used etching 
parameters, which are optimized to minimize the plasmainduced polymer deposition $\left(\mathrm{CF}_{4}-\mathrm{Ar} 56 \%-44 \%\right.$ at $10 \mathrm{sccm}$ with radiofrequency $(\mathrm{rf})$ power $=80 \mathrm{~W}$, dc bias $=350 \mathrm{~V}$, and pressure $=60-120 \mathrm{mTorr}$ ), and process duration (about $10 \mathrm{~min}$ ). It is found that a depth of imprinted features as low as a few tens of nanometers is enough in order to observe Braggscattering effects in organic gratings [23, 24]. The masters are then aligned manually with their features roughly parallel or perpendicular to the axis of a single light-emitting fiber, with precision better than $10^{\circ}$. Then, the masters are directly employed as molds for nanoimprinting without depositing any anti-sticking layer. RT-NIL is performed by a precision manual press (PW100 P/O/Weber, Germany) at room temperature, in air, with an applied force in the range $2.4-2.9 \mathrm{kN}$.

\subsection{Characterization}

Scanning electron microscopy (SEM) is performed using a Raith 150 electron-beam system operating with an acceleration voltage in the range 5-20 kV and an aperture size of 20-30 $\mu \mathrm{m}$. Atomic force microscopy (AFM) measurements are performed by a Veeco multimode head and a Nanoscope IIIa controller, in Tapping ${ }^{\mathrm{TM}}$ mode with $\mathrm{Si}$ cantilevers having a resonance frequency of $270 \mathrm{kHz}$.

Polarized PL spectroscopy is performed using the unpolarized light of a Hg lamp (U-RFL-T, Olympus, Tokyo, Japan) coupled to the $20 \times$ magnification objective of an inverted microscope (IX71, Olympus, Tokyo, Japan), allowing us to selectively excite a single fiber. The light emitted from each single fiber is collected by carefully coupling the PL into a fiber-connected monochromator (iHR320, HORIBA Jobin Yvon, Edison, NJ) equipped with a charge coupled device detector (Simphony CCD detection systems, HORIBA, Jobin Yvon). The polarization degree of the light emitted from each fiber is studied by collecting spectra from a rotating polarization analyzer. Finally, fluorescence micrographs of nanopatterned fibers are also collected by a confocal microscope (Olympus FV1000). The samples are excited by a diode laser (Nichia, Japan) emitting at $405 \mathrm{~nm}$, through a $40 \times$ objective lens with a numerical aperture (N.A.) of 0.85 .

\section{Results and discussion}

In a typical ES experiment, we prepare $0.7-1.0 \mathrm{mM}$ tetrahydrofuran solutions of F8BT. Optimized, beads-free and uniform light-emitting fibers are electrospun (inset of figure 2(a)) with diameters from about $100 \mathrm{~nm}$ to a few $\mu \mathrm{m}$ depending on the processing parameters, as investigated by SEM. The polarization of the emitted light is clearly appreciable by the spectra obtained by exciting the conjugated polymer fibers by unpolarized light and collecting fluorescence through a polarization analyzer (figure 2(a)). The individual F8BT fibers exhibit polarization ratios up to about 2.0. Figure 2(b) displays the normalized PL intensity as a function of the angle, $\theta$, between the orientation of the analyzer and the nanofiber longitudinal axis, and the corresponding fit to a Malus law, $I=I_{\|} \cos ^{2} \theta+I_{0}$, where $I_{0}$ indicates the intensity of the unpolarized background.
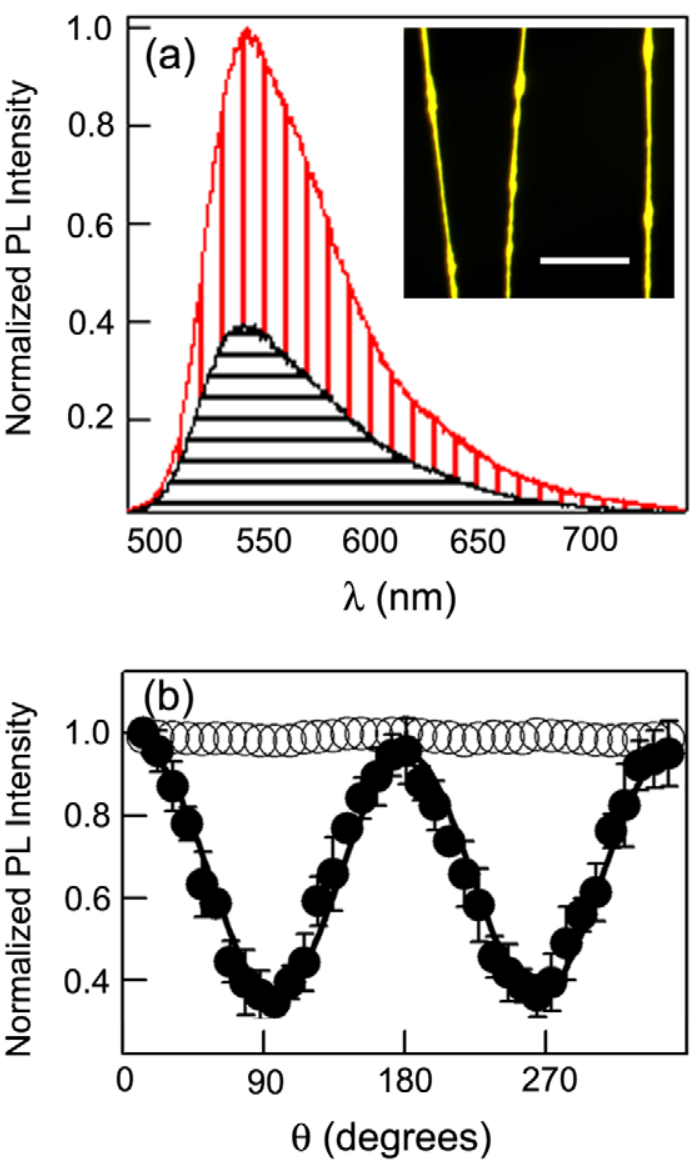

Figure 2. (a) PL spectra with analyzer parallel (vertically textured spectrum) and perpendicular (horizontally textured spectrum) to the nanofiber axis. Inset: fluorescence micrograph of the light-emitting F8BT fibers. Marker $=10 \mu \mathrm{m}$. (b) Dependence of the PL emission from conjugated polymer film (hollow circles) and from a single nanofiber (full circles) on the angle, $\theta$, between the polarizer and fiber axes. The solid line is a fit to the data by the Malus law, $I=I_{\|} \cos ^{2} \theta+I_{0}$, with $I_{\|}=(0.59 \pm 0.04)$ and $I_{0}=(0.04 \pm 0.02)$.

In order to enhance the PL polarization degree, we exploit RT-NIL to texture individual fibers by longitudinally oriented, imprinted features. Details on the nanopatterning procedure are reported elsewhere [16]. Fluorescent fibers with diameters intentionally produced to be in the $\mu \mathrm{m}$-scale are used for imprinting experiments. Master gratings having a period of $560 \mathrm{~nm}$ (figure 3(a)) are used for imprinting a single F8BT fiber at room temperature. This process is demonstrated to preserve the light-emitting properties of conjugated oligomers [23], polymers [24] and fluorescent dyes embedded in optically transparent matrices [25].

The planar views of the imprinted fiber, imaged by AFM, and its cross section, are shown in figures 3(b), (c) and in (d), respectively. We find that the RT-NIL allows the realization of patterns of very good quality on the top surface of deposited fibers, the grating faithfully reproducing the periodicity of the master. Moreover, differently from electrospun fibers imprinted with features orthogonally to their axis [16], we cannot appreciate any significant flattening upon RT-NIL, as evident by figure $3(\mathrm{~d})$, showing that the fiber body retains the same height and width in the pressed and untextured areas. 

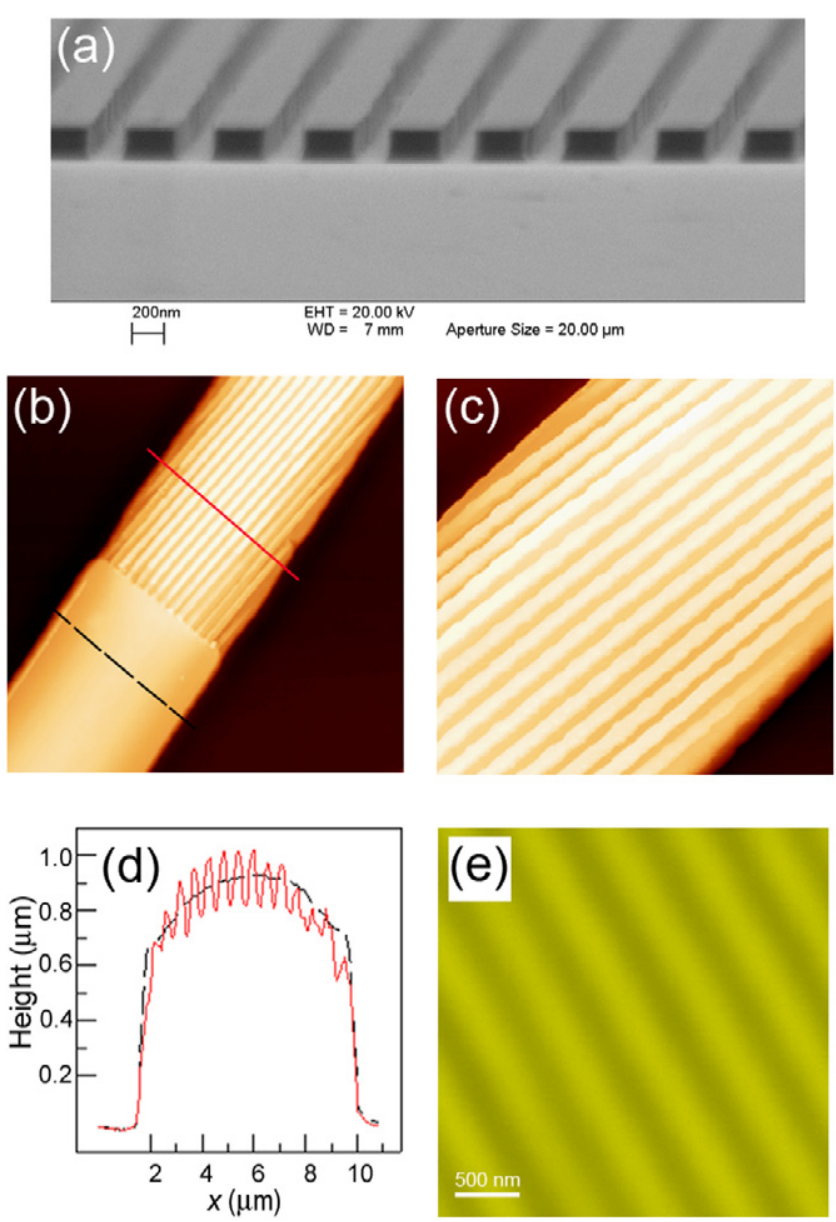

Figure 3. (a) Cross-sectional view of the master grating for RT-NIL. Grating period $560 \mathrm{~nm}$. (b), (c) AFM planar views of a longitudinally patterned F8BT fiber. Imaged area $=25 \times 25 \mu \mathrm{m}^{2}(\mathrm{~b})$ and $10 \times 10 \mu \mathrm{m}^{2}$ (c). Features period $=560 \mathrm{~nm}$. (d) Height profiles of unpatterned — black dashed line — and patterned — red solid line-regions of the fiber, corresponding to the dashed and continuous-marked cross-section in (b), respectively. (e) Confocal micrograph of nanoimprinted features on the surface of a light-emitting fiber.

Confocal microscopy (figure 3(e)) on nanopatterned fibers shows uniformly bright imprinted gratings.

The mechanical response of the conjugated polymer to the RT-NIL pressure is related to the viscoelastic behavior of the molecular backbones embedded in the fiber, and partially aligned by ES. Similarly to the imprinting of organic films, different contributions to the overall deformation are given by the conformational flexibility of single polymeric chains, which is mainly related to local molecular motions, and by the weakening of entanglements possibly formed between adjacent macromolecules, which is responsible for the longrange motion. In electrospun polymer nanofibers, structural intermolecular interactions can be reduced by the stretching induced by the applied field, thus resulting in shorter intervals needed for imprinting at room temperature with respect to the corresponding thin films, and more effective filling of the recessed features of the templates during printing. We carry out RT-NIL on individual F8BT nanofibers by using printing times as low as $5 \mathrm{~min}$, which are considerably shorter

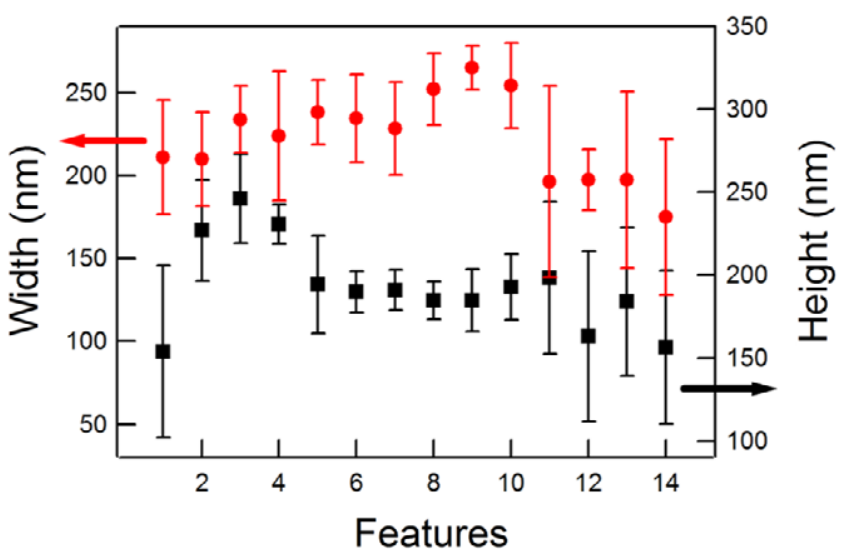

Figure 4. AFM measured height (black squares, right vertical scale) and width (red circles, left scale) of fiber imprinted features. The numbers on the horizontal axis (1-14) indicate the sequential order of parallel features (with period $560 \mathrm{~nm}$ ) on the fiber. Averages and standard deviations are calculated over ten measurements of each nanopatterned feature along the fiber axis.

than those employed for conjugated polymer films (up to a few hours) [24]. This also explains the absence of pressureinduced fiber flattening. In fact, this effect is intimately related to the larger amount (by about $25 \%$ as estimated by the achieved feature depth) of polymer flowing into the recessed features of the master, with respect to nanostructures printed with orientation perpendicular to the fiber longitudinal axis [16]. The here-patterned grooves exhibit an average depth of $(193 \pm 27) \mathrm{nm}$ and width of $(370 \pm 30) \mathrm{nm}$, well matching the features of the master (figures 3 and 4). Furthermore, we observe that a better and more uniform filling of the master nanochannels is accomplished in the axially central region with respect to the lateral edges, as estimated by the smaller standard deviations of the features' heights and widths (figure 4).

In RT-NIL, the stable pattern transfer is obtained by the irreversible deformation flow of the polymer, reaching its terminal flow region because of pressures quite a bit higher than values typical of conventional hot embossing. According to the Adams-Gibbs model [26], the polymer structural relaxation time, $\tau$, is determined by the configurational entropy, $S_{\mathrm{C}}$, which exhibits a dependence on pressure estimated by the molar thermal expansion of the amorphous system [27]. Consequently, the isothermal compression of the polymer system results in reducing the configurational entropy, which results in the excellent stability of the imprinted pattern [28]. Moreover, a remarkable role is usually played by the pressure-induced contraction of the free volume in the organic samples. The advantages over high-temperature lithography [29] are numerous, including the overall simplicity of the used setup, lower costs, and no heating of the active conjugated materials, in its turn determining wellpreserved light-emitting properties and processing capability in an uncontrolled atmosphere, together with the possible realization of crossed patterns by multilevel printing [24].

The influence of patterning on the polarization of the emission is displayed in figure 5(a). Here we plot the 

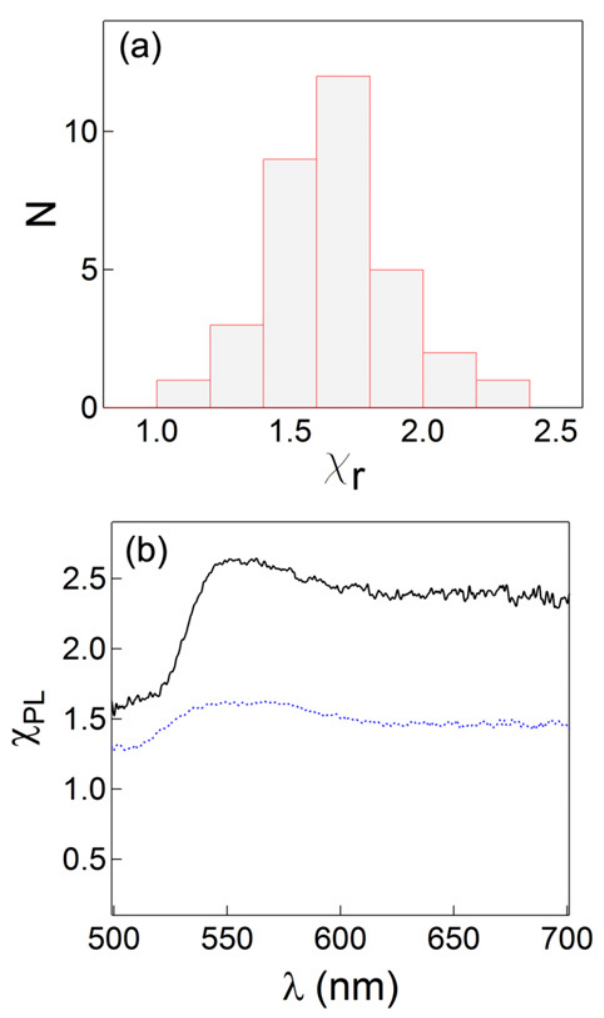

Figure 5. (a) Fibers distribution in terms of the patterning-induced increment of the PL polarization ratio, $\chi_{\mathrm{r}}$, calculated with respect to the $\chi_{\mathrm{PL}}$ value for as-spun single conjugated polymer fibers.

$N=$ number of fibers for each value of $\chi_{\mathrm{r}}$. Nanopatterned features-fiber axis angle $<10^{\circ}$. (b) Typical polarization ratio, $\chi_{\mathrm{PL}}$, versus wavelength, for an as-spun (dotted line) and a patterned (continuous line) F8BT fiber, respectively.

distribution of fibers in terms of $\chi_{\mathrm{r}}$, indicating the ratio between the values of $\chi_{\mathrm{PL}}$ measured in longitudinally nanopatterned and pristine form, respectively $\left(\chi_{\mathrm{r}}=\chi_{\mathrm{PL} \text {,patterned }} / \chi_{\mathrm{PL} \text {, unpatterned }}\right)$. We find that the fibers exhibit an enhancement of the polarization ratio up to a factor of 2.4 upon RT-NIL. The herereported values of $\chi_{\mathrm{r}}$ are obtained from polarization ratios calculated by integrated PL spectra intensities. Indeed, $\chi_{\mathrm{PL}}$ shows a spectral peak resembling the PL maximum both for as-spun and for patterned fibers, increasing upon increasing wavelength below the PL peak ( $\sim 550 \mathrm{~nm})$, and then showing a weak spectral dependence for wavelengths above the PL peak (figure 5(b)). This is physically expected given our unpolarized excitation scheme [30], leading to an almost constant value of $\chi_{\mathrm{r}}$ upon varying wavelength above the PL peak emission of F8BT. The slight reduction of the found optical anisotropy for photons of larger wavelength is in agreement with the occurrence of excitonic hopping processes within the fiber. Excitons whose emission results in light of longer wavelengths undergo a higher number of hopping events before radiative decay, thus losing a higher fraction of the original dipole moment orientation, as previously observed in other F8BT systems [30].

The polarization enhancement upon nanopatterning is related to two concomitant mechanisms, affecting the excitonic and the photonic nature of emission, respectively.

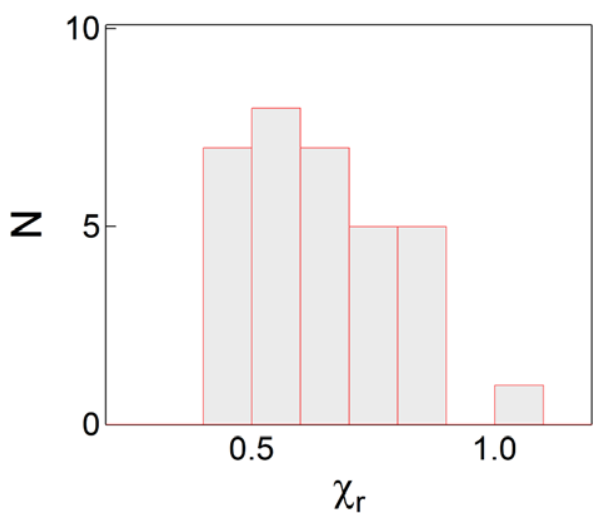

Figure 6. Distribution of the relative polarization ratio, $\chi_{\mathrm{r}}$, evidencing the lowering of PL polarization from single conjugated polymer fibers, upon patterning by RT-NIL with features perpendicular to the fiber axis. $N=$ number of fibers for each value of $\chi_{\mathrm{r}}$.

First, the nanoconfinement of the conjugated polymer chains within single imprinted features can result in an improved mutual alignment of the molecular backbones, as studied for self-organizing, liquid-crystalline luminescent polyfluorenes [30, 31]. As transition dipole moments are mostly parallel to the chain axis, this increases the optical anisotropy of the organic one-dimensional system. Second, linear distributed feedback microcavities with wavelengthscale period preferentially emit s-polarized light. Such freespace radiation with polarization parallel to the imprinted grooves corresponds to transversal electric modes within the Bragg structure [25, 32], which are guided perpendicularly to the axis of the longitudinally patterned fibers. Both mechanisms may contribute to increasing the polarization ratio of light emitted by textured fibers.

Aiming to assess the impact of the relative orientation between the fiber and the grating on the resulting polarization, we also pattern F8BT fibers by periodic features mainly perpendicular to the fiber axis by RT-NIL. In this case we find that the maximum polarization ratio from single fibers decreases by $50 \%$ (figure 6). In fact, such a nanopatterning relatively increases the forward light output from the fiber source, having an electrical field vector parallel to the grating grooves (transversal electric guided modes), besides possibly inducing a transversal nanoconfinement of conjugated polymer chains within single imprinted features, which would result in partial alignment of the transition moments perpendicularly with respect to the main fiber axis. This in turn determines an overall reduction of the axis-parallel polarized component of the emitted radiation.

In general, such imprinted gratings also contribute to significantly increase the external quantum efficiency of organic films [23, 24] or fibers [16], because of the enhanced Bragg-scattering towards some forward directions. In nanofibers, this is evidenced by angle-resolved PL measurements highlighting an enhancement of the forward emission, namely a reduction of the distance traveled by the emitted photons, and the consequent possible self-absorption, inside the organic medium [16]. 


\section{Conclusion}

In conclusion, we demonstrate room temperature nanopatterning of electrospun light-emitting fibers as an approach for enhancing the polarization of emitted light. Fibers formed from the prototype fluorene derivative, F8BT, imprinted with gratings with period matching the emission wavelength and features parallel to the fiber's longitudinal axis, exhibit an increase of the polarization ratio up to a factor of 2.4. The herefound PL polarization ratios, which are still about one order of magnitude lower than the values desirable for light-emitting device applications [33], could be further enhanced by using suitable composite materials, and by stretching after deposition [20]. These results fully extend the field of application of room temperature imprinting on active molecular materials in one-dimensional photonic nanostructures. Different photonic crystal geometries, realizable by multilevel RT-NIL starting from one-dimensional templates [24], will result in an in-depth control of the polarization state of emitted light.

\section{Acknowledgments}

We gratefully acknowledge Dr R Stabile and G Potente for the SEM investigations and fabrication of the masters. This work is partially supported by the Apulia Regional Strategic Project 'Ponamat' and the FIRB Contract RBIP06SH3W.

\section{References}

[1] Xia Y, Yang P, Sun Y, Wu Y, Mayers B, Yin Y, Kim F and Yan H 2003 Adv. Mater. 15 353-89

[2] Chabinyc M L, Chiu D T, Cooper McDonald J, Stroock A D, Christian J F, Karger A M and Whitesides G M 2001 Anal. Chem. 73 4491-8

[3] Pagliara S, Camposeo A, Polini A, Cingolani R and Pisignano D 2009 Lab Chip 9 2851-6

[4] Wang J, Gudiksen M S, Duan X, Cui Y and Lieber C M 2001 Science 293 1455-7

[5] O'Carroll D and Redmond G 2008 Physica E 40 2468-73

[6] Hu J, Li L-s, Yang W, Manna L, Wang L-W and Alivisatos A P 2001 Science 292 2060-3

[7] Grell M and Bradley D D C 1999 Adv. Mater. 11 895-905

[8] Jeukens C R L P N, Jonkheijm P, Wijnen F J P, Gielen J C, Christianen P C M, Schenning A P H J, Meijer E W and Maan J C 2005 J. Am. Chem. Soc. 127 8280-1
[9] Nguyen T-Q, Wu J, Doan V, Schwartz B J and Tolbert S H 2000 Science 288 652-6

[10] Reneker D H and Chun I 1996 Nanotechnology 7 216-23

[11] Li D, Wang Y and Xia Y 2003 Nano Lett. 3 1167-71

[12] Katta P, Alessandro M, Ramsier R D and Chase G G 2004 Nano Lett. 4 2215-8

[13] Kakade M V, Givens S, Gardner K, Lee K H, Chase D B and Rabolt J F 2007 J. Am. Chem. Soc. 129 2777-82

[14] Fennessey S F and Farris R J 2004 Polymer 45 4217-25

[15] Kwak G, Fukao S, Fujiki M, Sakaguchi T and Masuda T 2006 Chem. Mater. 18 5537-42

[16] Di Benedetto F, Camposeo A, Pagliara S, Mele E, Persano L, Stabile R, Cingolani R and Pisignano D 2008 Nat. Nanotechnol. 3 614-9

[17] Bianco A, Iardino G, Manuelli A, Bertarelli C and Zerbi G 2007 ChemPhysChem. 8 510-4

[18] Kongkhlang T, Tashiro K, Kotaki M and Chirachanchai S 2008 J. Am. Chem. Soc. 130 15460-6

[19] Bellan L M and Craighead H G 2008 Polymer 49 3125-9

[20] Campoy-Quiles M, Ishii Y, Sakai H and Murata H 2008 Appl. Phys. Lett. 92213305

[21] Kuo C-C, Wang C-T and Chen W-C 2008 Macromol. Mater. Eng. 293 999-1008

[22] Pagliara S, Camposeo A, Cingolani R and Pisignano D 2009 Appl. Phys. Lett. 95263301

[23] Pisignano D, Persano L, Raganato M F, Visconti P, Cingolani R, Barbarella G, Favaretto L and Gigli G 2004 Adv. Mater. 16 525-9

[24] Mele E, Di Benedetto F, Persano L, Cingolani R and Pisignano D 2005 Nano Lett. 5 1915-9

[25] Del Carro P, Camposeo A, Stabile R, Mele E, Persano L, Cingolani R and Pisignano D 2006 Appl. Phys. Lett. 89201105

[26] Adam G and Gibbs J H 1965 J. Chem. Phys. 43 139-46

[27] Casalini R, Capaccioli S, Lucchesi M, Rolla P A and Corezzi S 2001 Phys. Rev. E 63031207

[28] Mele E, Camposeo A, Stabile R, Del Carro P, Di Benedetto F, Persano L, Cingolani R and Pisignano D 2006 Appl. Phys. Lett. 89131109

[29] Sotomayor Torres C M (ed) 2003 Alternative Lithography (Boston, MA: Kluwer Academic)

[30] Schmid S A, Yim K H, Chang M H, Zheng Z, Huck W T S, Friend R H, Kim J S and Herz L M 2008 Phys. Rev. B 77115338

[31] Zheng Z, Yim K-H, Saifullah M S M, Welland M E, Friend R H, Kim J-S and Huck W T S 2007 Nano Lett. 7 987-92

[32] Turnbull G A, Andrew P, Jory M J, Barnes W L and Samuel I D W 2001 Phys. Rev. B 64125122

[33] Misaki M, Chikamatsu M, Yoshida Y, Azumi R, Tanigaki N, Yase K, Nagamatsu S and Ueda Y 2008 Appl. Phys. Lett. 93023304 